\title{
Disseminated Peritoneal Leiomyomatosis Following Previous Laparoscopic Myomectomy with Morcellation
}

\author{
Jae Hong Sang \\ Department of Obstetrics and Gynecology, Soonchunhyang University Bucheon Hospital, Soonchunhyang University College of Medicine, Bucheon, Korea
}

\begin{abstract}
Uterine leiomyomas are common benign neoplasms that develop from uterine muscle tissue, with an estimated incidence of 20\%$40 \%$ in women of reproductive age. Power morcellators are commonly used during myomectomy, but if all fragments are not removed, they may find another blood supply and develop into abdominal masses. We present a case of disseminated peritoneal leiomyomas in a patient with a history of laparoscopic myomectomy using an Endo-bag.
\end{abstract}

Keywords: Disseminated peritoneal leiomyomatosis; Laparoscopic myomectomy; Morcellation

\section{INTRODUCTION}

Uterine myomas are common benign tumors found in $20 \%-$ $40 \%$ of women of reproductive age, and they are subclassified by location. Disseminated peritoneal leiomyomatosis (DPL) is rare and can occur in patients after a myomectomy [1].

Intraperitoneal morcellation has a risk of leiomyosarcoma seeding during laparoscopic surgery performed before a biopsy for an accurate diagnosis. Leiomyosarcoma is found in 0.64 of 100,000 people, and the Food and Drug Administration (FDA) reported that an unknown uterine sarcoma was found in one of 350 women undergoing myomectomy or hysterectomy [2-4]. Specimens are extracted from the abdominal cavity using an Endo-bag to reduce the risk of intraperitoneal morcellation and the FDA recommended the use of a PneumoLiner for this in 2014.

We performed morcellation in a patient using an Endo-bag in laparoscopic myomectomy after a diagnosis of leiomyoma. Three years later, the patient suffered a relapse of leiomyoma and was diagnosed with DPL. We report a rare case of DPL in a patient with a history of laparoscopic myomectomy using an Endo-bag along with a literature review.

\section{CASE REPORT}

A 23-year-old nulliparous woman was transferred to Soonchunhyang University Bucheon Hospital for surgery for a $5.7-\mathrm{cm}$ myoma of the posterior uterine body found on sonography at a local clinic, after presenting with dysmenorrhea and menorrhagia. Blood tests including a cancer antigen 125 (CA125) of $20.1 \mathrm{IU} / \mathrm{mL}$ were normal.

In 2016, laparoscopic myomectomy with power morcellator was performed. A 6-cm intramural myoma was found in the posterior body of the uterus and the serosa was cut to excise it. After suturing the uterus, the myoma was removed in an Endo-bag after using a morcellator in the bag. After sufficient irrigation and suction of the pelvic cavity, no bleeding was seen and the operation was finished. A biopsy detected leiomyoma, but the patient was lost to follow-up.

In August 2019, she returned to the hospital with abdominal discomfort. Ultrasonography showed a relapse with multiple myomas. To relieve her symptoms, surgical treatment was offered. At that time, the patient's family history, risk factors, and blood tests were normal, including a CA125 of 29.7 IU/mL. A laparoscopic myomectomy was performed. During the operation, leiomyomas 

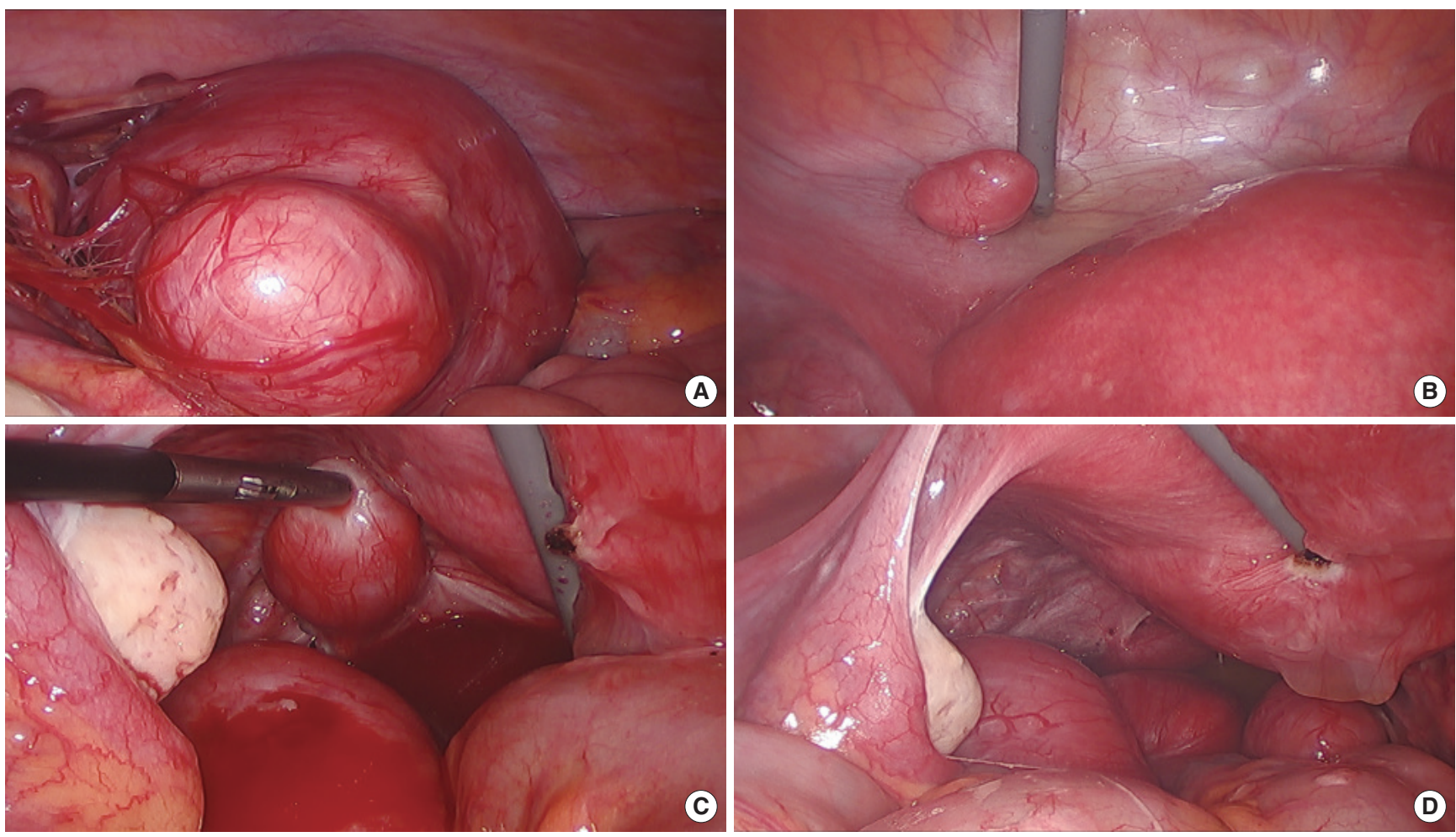

Fig. 1. (A-D) Multiple exophytic leiomyomas detected on the surface of omentum, peritoneum (included cul-de sac) during laparoscopic myomectomy.

ranging in size from 2 to $7 \mathrm{~cm}$ were found in the soft tissues, peritoneum, and omentum (Fig. 1). A myomectomy was performed for a suspected exophytic leiomyoma. A biopsy revealed leiomyoma. Subsequently, the patient has been followed regularly as an outpatient.

The patient provided written informed consent for the publication of clinical details and images.

\section{DISCUSSION}

Although uterine myoma is the most common benign tumor in women of reproductive age, peritoneal leiomyomatosis occurs rarely and the etiology and risk factors remain unclear. Myomectomies are commonly performed in gynecologic practice, but metastatic, but otherwise benign-appearing, leiomyomas are extremely rare. It is not clear why this rare subset of leiomyomas is prone to metastasis. They originate from uterine leiomyomas that are estrogen/progesterone-receptor positive and show inactivation of the second X chromosome [5].

Although the pathogenesis of metastatic leiomyomas is unclear, current hypotheses include lymphovascular spread, coelomic metaplasia, and detachment and revascularization of fibroids at distant sites [6]. The number of reported cases of DPL increased with the introduction of power morcellation. A recent systemic review found that $90 \%$ of cases of metastatic leiomyomas occurring after uterine surgery involved morcellation [7]. Therefore, intraperitoneal seeding during surgical intervention has been examined as a possible cause [2].

The iatrogenic spread of leiomyomas in the peritoneal cavity is of particular interest in light of recent concerns related to power morcellation in laparoscopic surgery. In a review of 600 laparoscopic gynecologic surgeries, Cucinella et al. [2] found that peritoneal myomas occurred in $1.2 \%$ of myomectomies using power morcellation. In their literature review of 13 studies, 93\% of DPLs occurred following laparoscopic procedures, specifically myomectomies, and a power morcellator was used in $87 \%$ of the cases. A more recent literature review of benign sequelae of unconfined power morcellation observed 200 cases and reported an incidence of DPL closer to $0.1 \%$ [8].

Our patient also had a relapse, although the morcellator was used in an Endo-bag during the initial laparoscopic myomectomy. We postulate that the relapse was likely caused by the overgrowth of 
smooth muscle cells and the remaining extracellular matrix (collagen, elastin, fibronectin, and proteoglycan) from myoma tissues left behind after the surgery.

We cannot purchase surgical equipment like the PneumoLiner in our country. Therefore, young nulliparous women in our country undergo laparotomy rather than laparoscopic surgery with a risk of DPL and leiomyosarcoma recurrence resulting from seeding.

\section{REFERENCES}

1. Ordulu Z, Dal Cin P, Chong WW, Choy KW, Lee C, Muto MG, et al. Disseminated peritoneal leiomyomatosis after laparoscopic supracervical hysterectomy with characteristic molecular cytogenetic findings of uterine leiomyoma. Genes Chromosomes Cancer 2010;49:1152-60.

2. Cucinella G, Granese R, Calagna G, Somigliana E, Perino A. Parasitic myomas after laparoscopic surgery: an emerging complication in the use of morcellator?: description of four cases. Fertil Steril 2011;96:e90-6.

3. Milad MP, Milad EA. Laparoscopic morcellator-related complications. J Minim Invasive Gynecol 2014;21:486-91.

4. George S, Barysauskas C, Serrano C, Oduyebo T, Rauh-Hain JA, Del Carmen MG, et al. Retrospective cohort study evaluating the impact of intraperitoneal morcellation on outcomes of localized uterine leiomyosarcoma. Cancer 2014;120:3154-8.

5. Miller J, Shoni M, Siegert C, Lebenthal A, Godleski J, McNamee C. Benign metastasizing leiomyomas to the lungs: an institutional case series and a review of the recent literature. Ann Thorac Surg 2016;101:253-8.

6. Awonuga AO, Shavell VI, Imudia AN, Rotas M, Diamond MP, Puscheck EE. Pathogenesis of benign metastasizing leiomyoma: a review. Obstet Gynecol Surv 2010;65:189-95.

7. Lete I, Gonzalez J, Ugarte L, Barbadillo N, Lapuente O, Alvarez-Sala J. Parasitic leiomyomas: a systematic review. Eur J Obstet Gynecol Reprod Biol 2016;203:250-9.

8. Tulandi T, Leung A, Jan N. Nonmalignant sequelae of unconfined morcellation at laparoscopic hysterectomy or myomectomy. J Minim Invasive Gynecol 2016;23:331-7. 